Bull. Korean Math. Soc. 50 (2013), No. 2, pp. 485-498

http://dx.doi.org/10.4134/BKMS.2013.50.2.485

\title{
SCHUR POWER CONVEXITY OF GINI MEANS
}

\author{
ZHEN-HANG YANG
}

AbStRaCt. In this paper, the Schur convexity is generalized to Schur $f$-convexity, which contains the Schur geometrical convexity, harmonic convexity and so on. When $f: \mathbb{R}_{+} \rightarrow \mathbb{R}$ is defined as $f(x)=\left(x^{m}-1\right) / m$ if $m \neq 0$ and $f(x)=\ln x$ if $m=0$, the necessary and sufficient conditions for $f$-convexity (is called Schur $m$-power convexity) of Gini means are given, which generalize and unify certain known results.

\section{Introduction}

Let $p, q \in \mathbb{R}$ and $a, b \in \mathbb{R}_{+}:=(0, \infty)$. The Gini means [13] are defined as

$$
G_{p, q}(a, b)= \begin{cases}\left(\frac{a^{p}+b^{p}}{a^{q}+b^{q}}\right)^{1 /(p-q)}, & p \neq q, \\ \exp \left(\frac{a^{p} \ln a+b^{p} \ln b}{a^{p}+b^{p}}\right), & p=q .\end{cases}
$$

It is easy to see that the Gini means $G_{p, q}(a, b)$ are continuous on the domain $\left\{(a, b ; p, q): a, b \in \mathbb{R}_{+} ; p, q \in \mathbb{R}\right\}$ and differentiable with respect to $(a, b) \in \mathbb{R}_{+}^{2}$ for fixed $p, q \in \mathbb{R}$. Also, Gini means are symmetric with respect to $a, b$ and $p, q$.

Gini means $G_{p, q}(a, b)$ contain many classical two variable means, for example, $G_{1,0}=A$ is the arithmetic mean, $G_{0,0}=G$ is the geometric mean, $G_{-1,0}=H$ is the harmonic mean, and more generally, the $p$-th power mean is equal to $G_{p, 0}, G_{p, p-1}$ is the Lehmer mean. The basic properties of Gini means, as well as their comparison theorems, log-convexities, and inequalities are studied in papers $[8,9,10,11,20,21,25,26,27,30,36,43,44,45,48]$.

Schur convexity was introduced by Schur in 1923 [22], and it has many important applications in analytic inequalities [2, 15, 49], linear regression [35], graphs and matrices [7], combinatorial optimization [16], information-theoretic topics [12], Gamma functions [23], stochastic orderings [32], reliability [17], and other related fields.

Received October 25, 2011.

2010 Mathematics Subject Classification. Primary 26B25, 26E60; Secondary 26D15.

Key words and phrases. Schur convexity, Schur power convexity, Gini means. 
In recent years, the Schur convexity and Schur geometrical convexity of $G_{p, q}(a, b)$ have attracted the attention of a considerable number of mathematicians $[4,5,19,29,28,31,33]$. Sándor [31] proved that the Gini means $G_{p, q}(a, b)$ are Schur convex on $(-\infty, 0] \times(-\infty, 0]$ and Schur concave on $[0, \infty) \times[0, \infty)$ with respect to $(p, q)$ for fixed $a, b>0$ with $a \neq b$. Yang [47] improved Sándor's result and proved that Gini means $G_{p, q}(a, b)$ are Schur convex with respect to $(p, q)$ for fixed $a, b>0$ with $a \neq b$ if and only if $p+q<0$ and Schur concave if and only if $p+q>0$. Wang and Zhang [38, 39] showed that Gini means $G_{p, q}(a, b)$ are Schur convex with respect to $(a, b) \in \mathbb{R}_{+}^{2}$ if and only if $p+q \geq 1$, $p, q \geq 0$ and Schur concave if and only if $p+q \leq 1, p \leq 0$ or $p+q \leq 1, q \leq 0$. $\mathrm{Gu}$ and Shi $[14,34]$ also discussed the Schur convexity. Recently, Chu and Xia [6] also proved the same result as Wang and Zhang's.

The Schur geometrical convexity was introduced by Zhang [50]. Wang and Zhang [39] proved Gini means $G_{p, q}(a, b)$ are Schur geometrically convex with respect to $(a, b) \in \mathbb{R}_{+}^{2}$ if $p+q \geq 0$ and Schur geometrically concave if $p+q \leq$ 0 . $\mathrm{Gu}$ and Shi $[14,34]$ also investigated the Schur geometrical convexities of Lehmer mean $G_{p, 1-p}(a, b)$ and Gini means $G_{p, q}(a, b)$, respectively.

Recently, Anderson et al. [1] discussed an attractive class of inequalities, which arise from the notion of harmonic convexity. And then it was started to research for Schur harmonic convexity. Chu et al. [3] showed that the Hamy symmetric function is Schur harmonic convex and obtained some analytic inequalities including the well-known Weierstrass inequalities. Xia [40] proved that the Lehmer mean $G_{p, p-1}(a, b)$ is Schur harmonic convex (Schur harmonic concave) with respect to $(a, b) \in \mathbb{R}_{+}^{2}$ if and only if $p \geq(\leq) 0$.

The purpose of this paper is to generalize the notion of Schur convexity and to investigate the so-called Schur power convexity of Gini means $G_{p, q}(a, b)$.

Our main results are as follows.

Theorem 1.1. For $m>0$ and fixed $(p, q) \in \mathbb{R}^{2}$, Gini mean $G_{p, q}(a, b)$ is Schur $m$-power convex with respect to $(a, b) \in \mathbb{R}_{+}^{2}$ if and only if $p+q \geq m$ and $\min (p, q) \geq 0$.

Theorem 1.2. For $m>0$ and fixed $(p, q) \in \mathbb{R}^{2}$, Gini mean $G_{p, q}(a, b)$ is Schur $m$-power concave with respect to $(a, b) \in \mathbb{R}_{+}^{2}$ if and only if $p+q \leq m$ and $\min (p, q) \leq 0$.

Theorem 1.3. For $m<0$ and fixed $(p, q) \in \mathbb{R}^{2}$, Gini mean $G_{p, q}(a, b)$ is Schur $m$-power convex with respect to $(a, b) \in \mathbb{R}_{+}^{2}$ if and only if $p+q \geq m$ and $\max (p, q) \geq 0$.

Theorem 1.4. For $m<0$ and fixed $(p, q) \in \mathbb{R}^{2}$, Gini mean $G_{p, q}(a, b)$ is Schur $m$-power concave with respect to $(a, b) \in \mathbb{R}_{+}^{2}$ if and only if $p+q \leq m$ and $\max (p, q) \leq 0$.

Theorem 1.5. For $m=0$ and fixed $(p, q) \in \mathbb{R}^{2}$, Gini mean $G_{p, q}(a, b)$ is Schur $m$-power convex (Schur $m$-power concave) with respect to $(a, b) \in \mathbb{R}_{+}^{2}$ if and only if $p+q \geq(\leq) 0$. 
The organization of the paper is as follows. In Section 2, based on the notions and lemmas of Schur convexity, we introduce the definition of Schur $f$-convex and Schur $f$-concave function, and prove the decision theorem for Schur $f$ convexity. As special case, the definition and decision theorem of Schur power convexity are deduced. In Section 3, some lemmas are given. In Section 4, our main results are proved.

\section{Schur $f$-convexity and Schur power convexity}

For convenience of readers, we recall some definitions as follows.

Definition $2.1([22,37])$. Let $\mathbf{x}=\left(x_{1}, x_{2}, \ldots, x_{n}\right)$ and $\mathbf{y}=\left(y_{1}, y_{2}, \ldots, y_{n}\right) \in$ $\mathbb{R}^{n}(n \geq 2)$.

(i) $\mathbf{x}$ is said to by majorized by $\mathbf{y}$ (in symbol $\mathbf{x} \prec \mathbf{y}$ ) if

$$
\sum_{i=1}^{k} x_{[i]} \leq \sum_{i=1}^{k} y_{[i]} \text { for } 1 \leq k \leq n-1, \quad \sum_{i=1}^{n} x_{[i]}=\sum_{i=1}^{n} y_{[i]},
$$

where $x_{[1]} \geq x_{[2]} \geq \cdots \geq x_{[n]}$ and $y_{[1]} \geq y_{[2]} \geq \cdots \geq y_{[n]}$ are rearrangements of $\mathbf{x}$ and $\mathbf{y}$ in a decreasing order.

(ii) $\mathbf{x} \geq \mathbf{y}$ means $x_{i} \geq y_{i}$ for all $i=1,2, \ldots, n$. Let $\Omega \subseteq \mathbb{R}^{n}(n \geq 2)$. The function $\phi: \Omega \rightarrow \mathbb{R}$ is said to be increasing if $\mathbf{x} \geq \mathbf{y}$ implies $\phi(\mathbf{x}) \geq \phi(\mathbf{y})$. $\phi$ is said to be decreasing if and only if $-\phi$ is increasing.

(iii) $\Omega \subseteq \mathbb{R}^{n}$ is called a convex set if $\left(\alpha x_{1}+\beta y_{1}, \ldots, \alpha x_{n}+\beta y_{n}\right) \in \Omega$ for all $\mathbf{x}, \mathbf{y}$ and all $\alpha, \beta \in[0,1]$ with $\alpha+\beta=1$.

(iv) Let $\Omega \subseteq \mathbb{R}^{n}(n \geq 2)$ be a set with nonempty interior. Then $\phi: \Omega \rightarrow \mathbb{R}$ is said to be Schur convex if $\mathbf{x} \prec \mathbf{y}$ on $\Omega$ implies $\phi(\mathbf{x}) \leq \phi(\mathbf{y})$. $\phi$ is said to be Schur concave if $-\phi$ is Schur convex.

Definition 2.2 ([22]). (i) $\Omega \subseteq \mathbb{R}^{n}(n \geq 2)$ is called a symmetric set, if $\mathbf{x} \in \Omega$ implies $\mathbf{x P} \in \Omega$ for every $n \times n$ permutation matrix $\mathbf{P}$.

(ii) The function $\phi: \Omega \rightarrow \mathbb{R}^{n}$ is called symmetric if for every permutation matrix $\mathbf{P}, \phi(\mathbf{x P})=\phi(\mathbf{x})$ for all $\mathbf{x} \in \Omega$.

For the Schur convexity, there is the following well-known result.

Lemma 2.1 ([22, 37]). Let $\Omega \subseteq \mathbb{R}^{n}$ be a symmetric set with nonempty interior $\Omega^{0}$ and $\phi: \Omega \rightarrow \mathbb{R}$ be continuous on $\Omega$ and differentiable in $\Omega^{0}$. Then $\phi$ is Schur convex (Schur concave) on $\Omega$ if and only if $\phi$ is symmetric on $\Omega$ and

$$
\left(x_{1}-x_{2}\right)\left(\frac{\partial \phi}{\partial x_{1}}-\frac{\partial \phi}{\partial x_{2}}\right) \geq(\leq) 0 \text {. }
$$

Next, let us define the Schur $f$-convexity as follows.

Definition 2.3. Let $\Omega=\mathbb{U}^{n}(\mathbb{U} \subseteq \mathbb{R})$ and $f$ be a strictly monotone function defined on $\mathbb{U}$. Assume that

$$
f(\mathbf{x})=\left(f\left(x_{1}\right), f\left(x_{2}\right), \ldots, f\left(x_{n}\right)\right) \text { and } f(\mathbf{y})=\left(f\left(y_{1}\right), f\left(y_{2}\right), \ldots, f\left(y_{n}\right)\right) .
$$


(i) $\Omega$ is called a $f$-convex set if $\left(f^{-1}\left(\alpha f\left(x_{1}\right)+\beta f\left(y_{1}\right)\right), \ldots, f^{-1}\left(\alpha f\left(x_{n}\right)+\right.\right.$ $\left.\left.\beta f\left(y_{n}\right)\right)\right) \in \Omega$ for all $\mathbf{x}, \mathbf{y} \in \Omega$ and all $\alpha, \beta \in[0,1]$ with $\alpha+\beta=1$.

(ii) Let $\Omega$ be a set with nonempty interior. Then function $\phi: \Omega \rightarrow \mathbb{R}$ is said to be Schur $f$-convex on $\Omega$ if $f(\mathbf{x}) \prec f(\mathbf{y})$ on $\Omega$ implies $\phi(\mathbf{x}) \leq \phi(\mathbf{y})$.

$\phi$ is said to be Schur $f$-concave if $-\phi$ is Schur $f$-convex.

Remark 2.1. Let $\Omega=\mathbb{U}^{n}(\mathbb{U} \subseteq \mathbb{R})$ and $f$ be a strictly monotone function defined on $\mathbb{U}$ and $f(\Omega)=\{f(\mathbf{x}): \mathbf{x} \in \Omega\}$. Then function $\phi: \Omega \rightarrow \mathbb{R}$ is Schur $f$-convex (Schur $f$-concave) if and only if $\phi \circ f^{-1}$ is Schur convex (Schur concave) on $f(\Omega)$.

Indeed, if function $\phi: \Omega \rightarrow \mathbb{R}$ is Schur $f$-convex, then $\forall \mathbf{x}^{\prime}, \mathbf{y}^{\prime} \in f(\Omega)$, there are $\mathbf{x}, \mathbf{y} \in \boldsymbol{\Omega}$ such that $\mathbf{x}^{\prime}=f(\mathbf{x}), \mathbf{y}^{\prime}=f(\mathbf{y})$. If $f(\mathbf{x}) \prec f(\mathbf{y})$, that is, $\mathbf{x}^{\prime} \prec \mathbf{y}^{\prime}$, then $\phi(\mathbf{x}) \leq \phi(\mathbf{y})$, that is, $\phi\left(\left(f^{-1}\left(\mathbf{x}^{\prime}\right)\right) \leq \phi\left(\left(f^{-1}\left(\mathbf{y}^{\prime}\right)\right)\right.\right.$. This shows that $\phi \circ f^{-1}$ is Schur convex on $f(\Omega)$. Conversely, if $\phi \circ f^{-1}$ is Schur convex on $f(\Omega)$, then $\forall \mathbf{x}, \mathbf{y} \in \Omega$ such that $f(\mathbf{x}) \prec f(\mathbf{y})$, we have $\phi\left(\left(f^{-1}(f(\mathbf{x}))\right) \leq \phi\left(\left(f^{-1}(f(\mathbf{y}))\right)\right.\right.$, that is, $\phi(\mathbf{x}) \leq \phi(\mathbf{y})$. This indicates $\phi$ is Schur $f$-convex on $\Omega$.

In the same way, we can show that $\phi$ is Schur $f$-concave on $\Omega$ if and only if $\phi \circ f^{-1}$ is Schur concave on $f(\Omega)$.

Remark 2.2. Let $\Omega \subseteq \mathbb{R}^{n}(n \geq 2)$ be a symmetric set and the function $\phi: \Omega \rightarrow \mathbb{R}$ be Schur $f$-convex (Schur $f$-concave). Then $\phi$ is symmetric on $\Omega$.

In fact, for any $\mathbf{x} \in \Omega$ and every permutation matrix $P$, we have $\mathbf{x} \mathbf{P} \in \Omega$. Note $\mathbf{x} \mathbf{P}$ is another permutation of $\mathbf{x}$, hence $f(\mathbf{x}) \prec f(\mathbf{x P}) \prec f(\mathbf{x})$. Since $\phi$ is Schur $f$-convex (Schur $f$-concave), we have $\phi(\mathbf{x}) \leq(\geq) \phi(\mathbf{x P}) \leq(\geq) \phi(\mathbf{x})$, that is, $\phi(\mathbf{x P})=\phi(\mathbf{x})$ for all $\mathbf{x} \in \Omega$. This shows that $\phi$ is symmetric on $\Omega$.

By Lemma 2.1 and Remarks 2.1, 2.2, we have the following:

Theorem 2.1. Assume that $\Omega=\mathbb{U}^{n}(\mathbb{U} \subseteq \mathbb{R})$ is a symmetric set with nonempty interior $\Omega^{0}, f$ is a strictly monotone and derivable function defined on $\mathbb{U}$, and $\phi: \Omega \rightarrow \mathbb{R}$ is continuous on $\Omega$ and differentiable in $\Omega^{0}$. Then $\phi$ is Schur $f$-convex (Schur $f$-concave) on $\Omega$ if and only if $\phi$ is symmetric on $\Omega$ and

$$
\left(f\left(x_{1}\right)-f\left(x_{2}\right)\right)\left(\frac{1}{f^{\prime}\left(x_{1}\right)} \frac{\partial \phi}{\partial x_{1}}-\frac{1}{f^{\prime}\left(x_{2}\right)} \frac{\partial \phi}{\partial x_{2}}\right) \geq(\leq) 0
$$

holds for any $\mathbf{x}=\left(x_{1}, x_{2}, \ldots, x_{n}\right) \in \Omega^{0}$ with $x_{1} \neq x_{2}$.

Proof. We easily check that $\phi \circ f^{-1}$ is symmetric on $f(\Omega)$ if and only if $\phi$ is symmetric on $\Omega$.

By Remark 2.1 and Lemma 2.1, $\phi \circ f^{-1}$ is Schur convex (Schur concave) if and only if $\phi \circ f^{-1}$ is symmetric on $f(\Omega)$ and

$$
\left(y_{1}-y_{2}\right)\left(\frac{\partial\left(\phi \circ f^{-1}\right)}{\partial y_{1}}-\frac{\partial\left(\phi \circ f^{-1}\right)}{\partial y_{2}}\right) \geq(\leq) 0
$$

holds for any $\mathbf{y} \in f(\Omega)^{0}$ with $y_{1} \neq y_{2}$. Substituting $f^{-1}(\mathbf{y})=\mathbf{x}$ yields $(2.3)$, where $\mathbf{x}=\left(x_{1}, x_{2}, \ldots, x_{n}\right) \in \Omega^{0}$ with $x_{1} \neq x_{2}$.

This proof is finished. 
Putting $f(x)=1, \ln x, x^{-1}$ in Definition 2.3 yield the Schur convexity, Schur geometrical convexity and Schur harmonic convexity. It is clear that the Schur $f$-convexity is a generalization of the Schur convexity mentioned above. In general, we have:

Definition 2.4. Let $f: \mathbb{R}_{+} \rightarrow \mathbb{R}$ be defined by $f(x)=\left(x^{m}-1\right) / m$ if $m \neq 0$ and $f(x)=\ln x$ if $m=0$. Then function $\phi: \Omega\left(\subseteq \mathbb{R}_{+}^{n}\right) \rightarrow \mathbb{R}$ is said to be Schur $m$-power convex on $\Omega$ if $f(\mathbf{x}) \prec f(\mathbf{y})$ on $\Omega$ implies $\phi(\mathbf{x}) \leq \phi(\mathbf{y})$.

$\phi$ is said to be Schur $m$-power concave if $-\phi$ is Schur $m$-power convex.

For the Schur power convexity, by Theorem 2.1 we have:

Corollary 2.1. Let $\Omega \subseteq \mathbb{R}_{+}^{n}$ be a symmetric set with nonempty interior $\Omega^{0}$ and $\phi: \Omega \rightarrow \mathbb{R}$ be continuous on $\Omega$ and differentiable in $\Omega^{0}$. Then $\phi$ is Schur $m$-power convex (Schur $m$-power concave) on $\Omega$ if and only if $\phi$ is symmetric on $\Omega$ and

$$
\begin{aligned}
\frac{x_{1}^{m}-x_{2}^{m}}{m}\left(x_{1}^{1-m} \frac{\partial \phi}{\partial x_{1}}-x_{2}^{1-m} \frac{\partial \phi}{\partial x_{2}}\right) & \geq(\leq) 0 \text { if } m \neq 0 \\
\left(\ln x_{1}-\ln x_{2}\right)\left(x_{1} \frac{\partial \phi}{\partial x_{1}}-x_{2} \frac{\partial \phi}{\partial x_{2}}\right) & \geq(\leq) 0 \text { if } m=0
\end{aligned}
$$

holds for any $\mathbf{x}=\left(x_{1}, x_{2}, \ldots, x_{n}\right) \in \Omega^{0}$ with $x_{1} \neq x_{2}$.

\section{Lemmas}

To prove the main results, we need the following useful lemmas.

Lemma 3.1. For fixed $(p, q) \in \mathbb{R}^{2}$, Gini means $G_{p, q}(a, b)$ is Schur m-power convex (Schur $m$-power concave) with respect to $(a, b) \in \mathbb{R}_{+}^{2}$ if and only if $g(t)$ $\geq(\leq) 0$ for all $t>0$, where

(3.1) $g(t):=g_{p, q}(t)= \begin{cases}\frac{(p-q) \sinh A t+p \sinh B t+q \sinh C t}{p-q} & \text { if } p \neq q, \\ \sinh (2 p-m) t-\sinh m t+2 p t \cosh m t & \text { if } p=q,\end{cases}$

and

$$
A=p+q-m, \quad B=p-q-m, \quad C=p-q+m .
$$

Proof. Let $m \neq 0$ and $G=G_{p, q}:=G_{p, q}(a, b)$ defined by (1.1).

For $p \neq q$, some simple partial derivative calculations yield

$$
\begin{aligned}
& \frac{\partial \ln G}{\partial a}=\frac{1}{G} \frac{\partial G}{\partial a}=\frac{1}{p-q}\left(\frac{p a^{p-1}}{a^{p}+b^{p}}-\frac{q a^{q-1}}{a^{q}+b^{q}}\right), \\
& \frac{\partial \ln G}{\partial b}=\frac{1}{G} \frac{\partial G}{\partial b}=\frac{1}{p-q}\left(\frac{p b^{p-1}}{a^{p}+b^{p}}-\frac{q b^{q-1}}{a^{q}+b^{q}}\right) .
\end{aligned}
$$

Therefore, we have

$$
a^{1-m} \frac{\partial \phi}{\partial a}-b^{1-m} \frac{\partial \phi}{\partial b}=\frac{G}{p-q}\left(p \frac{a^{p-m}-b^{p-m}}{a^{p}+b^{p}}-q \frac{a^{q-m}-b^{q-m}}{a^{q}+b^{q}}\right) .
$$


Substituting $\ln \sqrt{a / b}=t$ and using $\sinh x=\frac{1}{2}\left(e^{x}-e^{-x}\right), \cosh x=\frac{1}{2}\left(e^{x}+e^{-x}\right)$, the right hand side above can be written as

$$
\begin{aligned}
& a^{1-m} \frac{\partial \phi}{\partial a}-b^{1-m} \frac{\partial \phi}{\partial b} \\
= & \frac{G(a b)^{-m / 2}}{p-q}\left(p \frac{\sinh (p-m) t}{\cosh p t}-q \frac{\sinh (q-m) t}{\cosh q t}\right) \\
= & \frac{G(a b)^{-m / 2}}{2 \cosh p t \cosh q t} \frac{2 p \sinh (p-m) t \cosh q t-2 q \sinh (q-m) t \cosh p t}{p-q} .
\end{aligned}
$$

Using the "product into sum" formula for hyperbolic functions and (3.1), we have

$$
\begin{aligned}
\Delta & :=\frac{a^{m}-b^{m}}{m}\left(a^{1-m} \frac{\partial G_{p, q}}{\partial a}-b^{1-m} \frac{\partial G_{p, q}}{\partial b}\right) \\
& =\frac{a^{m}-b^{m}}{m(a-b)} \frac{(a-b) G_{p, q}}{2(a b)^{m / 2} \cosh p t \cosh q t} \frac{(p-q) \sinh A t+p \sinh B t+q \sinh C t}{p-q} \\
& =d_{p, q}(t) \cdot g_{p, q}(t),
\end{aligned}
$$

where

$$
d_{p, q}(t)=\frac{a^{m}-b^{m}}{m(a-b)} \frac{(a-b) G_{p, q}}{2(a b)^{m / 2} \cosh p t \cosh q t}(p \neq q)
$$

and $g_{p, q}(t)$ is defined by $(3.1)$.

In the case of $p=q$, since $G_{p, q}(a, b) \in C^{1}$ we have

$$
\frac{\partial G_{p, p}}{\partial a}=\lim _{q \rightarrow p} \frac{\partial G_{p, q}}{\partial a}, \quad \frac{\partial G_{p, p}}{\partial b}=\lim _{q \rightarrow p} \frac{\partial G_{p, q}}{\partial b} .
$$

It follows that

$$
\begin{aligned}
\Delta & =\frac{a^{m}-b^{m}}{m}\left(a^{1-m} \frac{\partial G_{p, p}}{\partial a}-b^{1-m} \frac{\partial G_{p, p}}{\partial b}\right) \\
& =\lim _{q \rightarrow p}\left(\frac{a^{m}-b^{m}}{m}\left(a^{1-m} \frac{\partial G_{p, q}}{\partial a}-b^{1-m} \frac{\partial G_{p, q}}{\partial b}\right)\right) \\
& =\lim _{q \rightarrow p}\left(d_{p, q}(t) g_{p, q}(t)\right)=g_{p, p}(t) \lim _{q \rightarrow p} d_{p, q}(t) .
\end{aligned}
$$

Summarizing two cases above yield

$$
\begin{aligned}
& \Delta=\frac{a^{m}-b^{m}}{m}\left(a^{1-m} \frac{\partial \phi}{\partial a}-b^{1-m} \frac{\partial \phi}{\partial b}\right) \\
& =\left\{\begin{array}{cl}
g_{p, q}(t) \cdot d_{p, q}(t) & \text { if } p \neq q, \\
g_{p, p}(t) \lim _{q \rightarrow p} d_{p, q}(t) & \text { if } p=q .
\end{array}\right.
\end{aligned}
$$

Since $\Delta$ is symmetric with respect to $a$ and $b$, without loss of generality we assume $a>b$. It is easy to verify that $\frac{a^{m}-b^{m}}{m(a-b)}>0, \frac{(a-b) G_{p, q}}{2(a b)^{m / 2}}>0$, and $\frac{1}{\cosh p t \cosh q t}>0$ for $t=\ln \sqrt{a / b}>0$, which implies that $d_{p, q}(t)$ and its limit at 
$p=q$ are both positive. Thus by Corollary 2.1 Gini mean $G_{p, q}(a, b)$ is Schur $m$-power convex (Schur $m$-power concave) with respect to $(a, b) \in \mathbb{R}_{+}^{2}$ if and only if $\Delta \geq(\leq) 0$ if and only if $g(t)=g_{p, q}(t) \geq(\leq) 0$ for all $t>0$.

It is easy to check that for $m=0$ this lemma is also true.

This lemma is proved.

Lemma 3.2. Let $g(t)=g_{p, q}(t)$ be defined by (3.1). Then

$$
\lim _{t \rightarrow 0, t>0} \frac{g_{p, q}(t)}{2 t}=p+q-m \text {. }
$$

Proof. It is easy to check that $g(0)=0$.

In the case of $p \neq q$, applying L'Hospital's rule yields

$$
\begin{aligned}
\lim _{t \rightarrow 0, t>0} \frac{g_{p, q}(t)}{2 t} & =\lim _{t \rightarrow 0, t>0} \frac{\partial g_{p, q}(t)}{2 \partial t} \\
& =\frac{(p-q) A+p B+q C}{2(p-q)}=p+q-m .
\end{aligned}
$$

In the case of $p=q$, we have

$$
\lim _{t \rightarrow 0, t>0} \frac{g_{p, p}(t)}{2 t}=2 p-m .
$$

This completes the proof.

Lemma 3.3. Let $m>0$ and $\beta=\max (|A|,|B|,|C|)$ where $A, B, C$ are defined by (3.1). Then

(i) if $p>q$, then

$$
\lim _{t \rightarrow \infty} \frac{2 \beta g_{p, q}(t)}{e^{\beta t}}= \begin{cases}p+q-m & \text { if } p>q>m \text { or } 0>p>q \\ \frac{p^{2}}{p-m} & \text { if } p>q=m \\ 2(q-m) & \text { if } p=0>q \\ \frac{q(p-q+m)}{p-q} & \text { if } p>0, q<m, p>q\end{cases}
$$

(ii) if $p=q$, then

$$
\lim _{t \rightarrow \infty} \frac{2 \beta g_{p, p}(t)}{e^{\beta t}}= \begin{cases}2 p-m & \text { if } p>m \text { or } p<0 \\ -2 m & \text { if } p=0 \\ \infty & \text { if } 0<p \leq m .\end{cases}
$$

Proof. (3.4)-(3.5) easily follows from the following limit relations:

$$
\begin{aligned}
\lim _{t \rightarrow \infty} \frac{2 \cosh \alpha t}{e^{\beta t}} & = \begin{cases}1 & \text { if } \beta=|\alpha|, \\
0 & \text { if } \beta>|\alpha|,\end{cases} \\
\lim _{t \rightarrow \infty} \frac{2 \alpha t \sinh \alpha t}{e^{\beta t}} & = \begin{cases}\infty & \text { if } \beta=|\alpha|, \\
0 & \text { if } \beta>|\alpha| .\end{cases}
\end{aligned}
$$

(i) If $p>q$, then $\beta=\max (|A|,|B|,|C|)=\max (|A|,|C|)$ because $|C|^{2}-$ $|B|^{2}=4 m(p-q)>0$. We have

$$
(p-q) \lim _{t \rightarrow \infty} \frac{2 \beta g_{p, q}(t)}{e^{\beta t}}=(p-q) \lim _{t \rightarrow \infty} \frac{2}{e^{\beta t}} \frac{\partial g_{p, q}(t)}{\partial t}
$$




$$
\begin{aligned}
& =\lim _{t \rightarrow \infty} 2 \frac{(p-q) A \cosh A t+p B \cosh B t+q C \cosh C t}{e^{\beta t}} \\
& = \begin{cases}(p-q) A & \text { if }|A|>|C|, \text { i.e., } p(q-m)>0, \\
(p-q) A+q C & \text { if }|A|=|C| \text {, i.e., } p(q-m)=0, \\
q C & \text { if }|A|<|C| \text {, i.e., } p(q-m)<0 .\end{cases} \\
& = \begin{cases}(p-q)(p+q-m) & \text { if } p>q>m \text { or } 0>p>q, \\
p^{2} & \text { if } p>q=m, \\
-2 q(q-m) & \text { if } p=0>q, \\
q(p-q+m) & \text { if } p>0, q<m, p>q .\end{cases}
\end{aligned}
$$

Dividing by $(p-q)$ in the above limit relation yields (3.4).

(ii) If $p=q$, then $\beta=\max (|A|,|B|,|C|)=\max (|2 p-m|, m)$. We have

$$
\begin{aligned}
& \lim _{t \rightarrow \infty} \frac{2 \beta g_{p, p}(t)}{e^{\beta t}}=\lim _{t \rightarrow \infty} \frac{2}{e^{\beta t}} \frac{\partial g_{p, p}(t)}{\partial t} \\
= & \lim _{t \rightarrow \infty} 2 \frac{(2 p-m) \cosh (2 p-m) t+(2 p-m) \cosh m t+2 m p \sinh m t}{e^{\beta t}} \\
= & \begin{cases}2 p-m & \text { if }|2 p-m|>m, \text { i.e., } p>m \text { or } p<0, \\
\infty & \text { if }|2 p-m|=m, p \neq 0, \text { i.e., } p=m, \\
-2 m & \text { if }|2 p-m|=m, p=0, \text { i.e. } p=0, \\
\infty & \text { if }|2 p-m|<m, \text { i.e., } 0<p<m,\end{cases}
\end{aligned}
$$

which implies (3.5).

This completes the proof.

\section{Proof of main results}

Proof of Theorem 1.1. Assume that

$$
E_{1}=\{(p, q): p+q-m \geq 0, \min (p, q) \geq 0\} \quad(m>0) .
$$

By Lemma 3.1, to prove Theorem 1.1, it suffices to prove that $g_{p, q}(t) \geq 0$ for all $t>0$ if and only if $(p, q) \in E_{1}$.

Necessity. We prove that $(p, q) \in E_{1}$ is the necessary conditions for $g(t)=$ $g_{p, q}(t) \geq 0$ for all $t>0$. It is obvious that

$$
\lim _{t \rightarrow 0, t>0} \frac{g_{p, q}(t)}{2 t} \geq 0 \text { and } \lim _{t \rightarrow \infty} \frac{2 \beta g_{p, q}(t)}{e^{\beta t}} \geq 0 .
$$

Now, we get the necessary conditions from (4.1) together with (3.4) and (3.5). To this aim, we distinguish three cases.

(i) Case 1: $p>q$. By (4.1) together with (3.3) and (3.4), we have

Subcase 1:

$$
\left\{\begin{array}{l}
p+q-m \geq 0 \\
p+q-m \geq 0, \\
p>q>m \text { or } 0>p>q
\end{array} \quad \Longrightarrow p>q>m\right.
$$

which implies $(p, q) \in\{(p, q): p>q>m\}:=E_{11}$. 


\section{Subcase 2:}

$$
\left\{\begin{array}{l}
p+q-m \geq 0, \\
\frac{p^{2}}{p-m} \geq 0, \\
p>q=m
\end{array} \Longrightarrow p>q=m\right.
$$

which implies $(p, q) \in\{(p, q): p>q=m\}:=E_{12}$.

Subcase 3:

Subcase 4:

$$
\left\{\begin{array}{l}
p+q-m \geq 0 \\
2(q-m) \geq 0, \\
p=0>q
\end{array} \Longrightarrow\right. \text { which is impossible. }
$$

$$
\left\{\begin{array} { l } 
{ p + q - m \geq 0 , } \\
{ \frac { q ( p - q + m ) } { p - q } \geq 0 , } \\
{ p > 0 , } \\
{ q < m , } \\
{ p > q }
\end{array} \Longrightarrow \left\{\begin{array}{l}
p+q-m \geq 0 \\
p>0 \\
0<q<m \\
p>q
\end{array}\right.\right.
$$

which implies $(p, q) \in\{(p, q): p+q-m \geq 0, p>0,0<q<m, p>q\}:=E_{14}$.

$\left(\mathbf{i}^{\prime}\right)$ Case $1^{\prime}: p<q$. Since $g_{p, q}(t)$ is symmetric with respect to $p$ and $q$, we get $(p, q) \in E_{111}^{\prime} \cup E_{112}^{\prime} \cup E_{114}^{\prime}$, where

$$
\begin{aligned}
& E_{11}^{\prime}=\{(p, q): q>p>m\}, E_{12}^{\prime}=\{(p, q): q>p=m\}, \\
& E_{14}^{\prime}=\{(p, q): p+q-m \geq 0, q>0,0<p<m, q>p\} .
\end{aligned}
$$

(ii) Case 2: $p=q$. By (4.1) together with (3.3) and (3.5), we have Subcase 1:

\section{Subcase 2:}

$$
\left\{\begin{array}{l}
p+q-m \geq 0 \\
2 p-m \geq 0 \\
p>m \text { or } p<0
\end{array} \Longrightarrow p=q>m\right.
$$

$$
\left\{\begin{array}{l}
p+q-m \geq 0, \\
-2 m \geq 0, \\
p=0
\end{array} \Longrightarrow\right. \text { which is impossible. }
$$

Subcase 3:

$$
\left\{\begin{array}{l}
p+q-m \geq 0, \\
\infty \geq 0, \\
0<p \leq m
\end{array} \Longrightarrow \frac{m}{2} \leq p=q<m .\right.
$$

The above three subcases imply $(p, q) \in\left\{(p, q): p=q \geq \frac{m}{2}\right\}:=E_{10}$.

Summarizing all the cases (i), (i') and (ii) yields

$$
(p, q) \in\left(E_{11} \cup E_{12} \cup E_{14}\right) \cup\left(E_{11}^{\prime} \cup E_{12}^{\prime} \cup E_{14}^{\prime}\right) \cup E_{10}=E_{1} .
$$

Sufficiency. We prove the condition $(p, q) \in E_{1}$ is sufficient for $g(t)=g_{p, q}(t) \geq$ 0 for all $t>0$. Since $g(0)=0$, it is enough to prove $g^{\prime}(t) \geq 0$ if $(p, q) \in E_{1}$. For symmetry, we may assume again that $p \geq q$. 
Noting

we have

$$
(p-q) A=p B+q C \text { or } p B=(p-q) A-q C,
$$

$$
\begin{aligned}
(p-q) g^{\prime}(t) & =(p-q) A \cosh A t+p B \cosh B t+q C \cosh C t \\
& =(p-q) A(\cosh A t+\cosh B t)+q C(\cosh C t-\cosh B t) \\
& =(p-q) A(\cosh A t+\cosh B t)+2 q C \sinh (p-q) t \sinh m t .
\end{aligned}
$$

If $p>q$ and $(p, q) \in E_{1}$, then $A=p+q-m \geq 0, q=\min (p, q) \geq 0$, $C=p-q+m>0$. It follows that $(p-q) g^{\prime}(t) \geq 0$ for $(p, q) \in E_{1}$.

If $p=q$ and $(p, q) \in E_{1}$, then $2 p-m \geq 0, p=\min (p, q) \geq 0$. Therefore,

(4.3) $g^{\prime}(t)=(2 p-m) \cosh (2 p-m) t+(2 p-m) \cosh m t+2 m p \sinh m t \geq 0$.

This completes the proof of Theorem 1.1.

Proof of Theorem 1.2. Assume that

$$
\begin{aligned}
& E_{2}=\{(p, q\}: p+q-m \leq 0, p \geq q, q \leq 0\} \quad(m>0), \\
& E_{2}^{\prime}=\{(p, q\}: p+q-m \leq 0, q \geq p, p \leq 0\} \quad(m>0),
\end{aligned}
$$

then

$$
E_{2} \cup E_{2}^{\prime}=\{(p, q\}: p+q-m \leq 0 \text { and } \min (p, q) \leq 0\} \quad(m>0) .
$$

By Lemma 3.1, to prove Theorem 1.2, it suffices to show that $g_{p, q}(t) \leq 0$ for all $t>0$ if and only if $(p, q) \in E_{2} \cup E_{2}^{\prime}$.

Necessity. If $g_{p, q}(t) \leq 0$ for all $t>0$, then

$$
\lim _{t \rightarrow 0, t>0} \frac{g_{p, q}(t)}{2 t} \leq 0 \text { and } \lim _{t \rightarrow \infty} \frac{2 \beta g_{p, q}(t)}{e^{\beta t}} \leq 0 .
$$

Similarly, we divide the proof of necessity into three cases.

(i) Case 1: $p>q$. By (4.4) together with (3.3) and (3.4), we have

Subcase 1:

$$
\left\{\begin{array}{l}
p+q-m \leq 0, \\
p+q-m \leq 0, \\
p>q>m \text { or } 0>p>q
\end{array} \Longrightarrow 0>p>q,\right.
$$

which implies $(p, q) \in\{(p, q): 0>p>q\}:=E_{21}$.

Subcase 2:

Subcase 3:

$$
\left\{\begin{array}{l}
p+q-m \leq 0 \\
\frac{p^{2}}{p-m} \leq 0 \\
p>q=m
\end{array} \Longrightarrow\right. \text { which is impossible. }
$$

$\left\{\begin{array}{c}p+q-m \leq 0, \\ 2(q-m) \leq 0, \\ p=0>q\end{array} \Longrightarrow p=0>q\right.$,

which implies $(p, q) \in\{(p, q): p=0>q\}:=E_{23}$. 


\section{Subcase 4:}

$$
\left\{\begin{array} { l } 
{ p + q - m \leq 0 } \\
{ \frac { q ( p - q + m ) } { p - q } \leq 0 , } \\
{ p > 0 } \\
{ q < m } \\
{ p > q }
\end{array} \Longrightarrow \left\{\begin{array}{c}
p+q-m \leq 0 \\
p>0 \geq q
\end{array}\right.\right.
$$

which implies $(p, q) \in\{(p, q): p+q-m \leq 0, p>0 \geq q\}:=E_{24}$.

$\left(\mathrm{i}^{\prime}\right)$ Case $1^{\prime}: p<q$. Since $g_{p, q}(t)$ is symmetric with respect to $p$ and $q$, so $(p, q) \in E_{21}^{\prime} \cup E_{23}^{\prime} \cup E_{24}^{\prime}$, where

$$
\begin{aligned}
& E_{21}^{\prime}=\{(p, q): 0>q>p\}, \\
& E_{23}^{\prime}=\{(p, q): q=0>p\}, \\
& E_{24}^{\prime}=\{(p, q): p+q-m \leq 0, q>0 \geq p\} .
\end{aligned}
$$

(ii) Case 2: $p=q$. By (4.4) together with (3.3) and (3.5), we have Subcase 1:

\section{Subcase 2:}

$$
\left\{\begin{array}{l}
p+q-m \leq 0 \\
2 p-m \leq 0 \\
p>m \text { or } p<0
\end{array} \Longrightarrow p=q<0\right.
$$

\section{Subcase 3:}

$$
\left\{\begin{array}{l}
p+q-m \leq 0, \\
-2 m \leq 0 \\
p=0
\end{array} \Longrightarrow p=q=0\right.
$$

$$
\left\{\begin{array}{l}
p+q-m \leq 0, \\
\infty \leq 0, \\
0<p \leq m
\end{array} \Longrightarrow\right. \text { which is impossible. }
$$

The above three subcases imply $(p, q) \in\{(p, q): p=q \leq 0\}:=E_{20}$. Summarizing all the cases (i), (i') and (ii) yields

$$
(p, q) \in\left(E_{21} \cup E_{23} \cup E_{24}\right) \cup\left(E_{21}^{\prime} \cup E_{23}^{\prime} \cup E_{24}^{\prime}\right) \cup E_{20}=E_{2} \cup E_{2}^{\prime} .
$$

Sufficiency. Similarly to proof of sufficiency of Theorem 1.1, by (4.2) and (4.3) we easily prove $g^{\prime}(t) \leq 0$ if $(p, q) \in E_{2} \cup E_{2}^{\prime}$. Hence $g_{p, q}(t)=g(t) \leq g(0)=0$ for all $t>0$.

The proof of Theorem 1.2 is completed.

Proof of Theorem 1.3. Let $g_{p, q, m}(t):=g_{p, q}(t)$ be defined by (3.1) and

$$
p^{\prime}=-p, \quad q^{\prime}=-q, \quad m^{\prime}=-m .
$$

We easily verify that, for $p, q, p^{\prime}, q^{\prime}, m, m^{\prime} \in \mathbb{R}$,

$$
g_{p, q, m}(t)=-g_{p \prime, q \prime, m \prime}(t) .
$$


From this and Lemma 3.1, for $m<0$, Gini mean $G_{p, q}(a, b)$ is Schur $m$-power convex if and only if $G_{p \prime, q}(a, b)$ is Schur $m^{\prime}$-power concave with respect to $(a, b) \in \mathbb{R}_{+}^{2}$, which, by Theorem 1.2 , if and only if

$$
p^{\prime}+q^{\prime} \leq m^{\prime} \text { and } \min \left(p^{\prime}, q^{\prime}\right) \leq 0,
$$

that is,

$$
p+q \geq m \text { and } \max (p, q) \geq 0 .
$$

Theorem 1.3 follows.

Proof of Theorem 1.4. Similarly as in the proof of Theorem 1.3, for $m<0$, Gini mean $G_{p, q}(a, b)$ is Schur $m$-power concave if and only if $G_{p \prime, q}(a, b)$ is Schur $m^{\prime}$-power convex with respect to $(a, b) \in \mathbb{R}_{+}^{2}$, which, by Theorem 1.1 , if and only if

$$
p^{\prime}+q^{\prime} \geq m^{\prime} \text { and } \min \left(p^{\prime}, q^{\prime}\right) \geq 0,
$$

that is,

$$
p+q \leq m \text { and } \max (p, q) \leq 0,
$$

The proof of Theorem 1.4 ends.

Proof of Theorem 1.5. By Lemma 3.1, to prove Theorem 1.5, it is enough to prove that $g_{p, q}(t) \geq(\leq) 0$ for all $t>0$ if and only if $p+q \geq(\leq) 0$ for $m=0$. To this end, we divide the proof into two cases.

(i) Case 1: $p \neq q$. By (3.1), we have

$$
\begin{aligned}
g_{p, q}(t) & =\frac{(p-q) \sinh (p+q) t+(p+q) \sinh (p-q) t}{p-q} \\
& = \begin{cases}t(p+q)\left(\frac{\sinh (p+q) t}{(p+q) t}+\frac{\sinh (p-q) t}{(p-q) t}\right) & \text { if } p+q \neq 0, \\
0 & \text { if } p+q=0 .\end{cases}
\end{aligned}
$$

Since $\frac{\sinh u}{u}>0$ for all $u \neq 0$ and $t>0$, we obtain $\operatorname{sgn}\left(g_{p, q}(t)\right)=\operatorname{sgn}(p+q)$.

(ii) Case 2: $p=q$. By (3.1), we have

$$
g_{p, p}(t)= \begin{cases}2 p t\left(\frac{\sinh (2 p t)}{2 p t}+1\right) & \text { if } p \neq 0 \\ 0 & \text { if } p=0\end{cases}
$$

It is obvious that $\operatorname{sgn}\left(g_{p, p}(t)\right)=\operatorname{sgn}(p)$.

In brief, $g_{p, q}(t) \geq(\leq) 0$ for all $t>0$ if and only if $p+q \geq(\leq) 0$.

The proof of Theorem 1.5 is finished.

\section{References}

[1] G. D. Anderson, M. K. Vamanamurthy, and M. Vuorinen, Generalized convexity and inequalities, J. Math. Anal. Appl. 335 (2007), no. 2, 1294-1308.

[2] J. S. Aujla and F. C. Silva, Weak majorization inequalities and convex functions, Linear Algebra Appl. 369 (2003), 217-233.

[3] Y. M. Chu and Y.-P. Lv, The Schur harmonic convexity of the Hamy symmetric function and its applications, J. Inequal. Appl. 2009 (2009), Art. ID 838529, 10 pages. 
[4] Y. M. Chu and X. M. Zhang, Necessary and sufficient conditions such that extended mean values are Schur-convex or Schur-concave, J. Math. Kyoto Univ. 48 (2008), no. $1,229-238$.

[5] Y. M. Chu, X. M. Zhang, and G.-D. Wang, The Schur geometrical convexity of the extended mean values, J. Convex Anal. 15 (2008), no. 4, 707-718.

[6] Y. M. Chu and W. F. Xia, Solution of an open problem for Schur convexity or concavity of the Gini mean values, Sci. China Ser. A 52 (2009), no. 10, 2099-2106.

[7] G. M. Constantine, Schur convex functions on the spectra of graphs, Discrete Math. 45 (1983), no. 2-3, 181-188.

[8] P. Czinder and Zs. Páles, A general Minkowski-type inequality for two variable Gini means, Publ. Math. Debrecen 57 (2000), no. 1-2, 203-216.

[9] Local monotonicity properties of two-variable Gini means and the comparison theorem revisited, J. Math. Anal. Appl. 301 (2005), no. 2, 427-438.

[10] Z. Daróczy and L. Losonczi, Über den Vergleich von Mittelwerten, Publ. Math. Debrecen 17 (1970), 289-297.

[11] D. Farnsworth and R. Orr, Gini means, Amer. Math. Monthly 93 (1986), no. 8, 603-607.

[12] A. Forcina and A. Giovagnoli, Homogeneity indices and Schur-convex functions, Statistica 42 (1982), no. 4, 529-542.

[13] C. Gini, Diuna formula comprensiva delle media, Metron 13 (1938), 3-22.

[14] Ch. Gu and H. N. Shi, Schur-convexity and Schur-geometric convexity of Lehmer means, Math. Prac. Theory 39 (2009), no. 12, 183-188.

[15] G. H. Hardy, J. E. Littlewood, and G. Pólya, Some simple inequalities satisfied by convex functions, Messenger Math. 58 (1929), 145-152.

[16] F. K. Hwang and U. G. Rothblum, Partition-optimization with Schur convex sum objective functions, SIAM J. Discrete Math. 18 (2004), no. 3, 512-524.

[17] F. K. Hwang, U. G. Rothblum, and L. Shepp, Monotone optimal multipartitions using Schur convexity with respect to partial orders, SIAM J. Discrete Math. 6 (1993), no. 4, 533-547.

[18] D.-M. Li, Ch. Gu, and H.-N. Shi, Schur convexity of the power-type generalization of Heronian mean, Math. Prac. Theory 36 (2006), no. 9, 387-390.

[19] D.-M. Li and H.-N. Shi, Schur convexity and Schur-geometrically concavity of generalized exponent mean, J. Math. Inequal. 3 (2009), no. 2, 217-225.

[20] Zh. Liu, Minkowski's inequality for extended mean values, Proceedings of the Second ISAAC Congress, Vol. 1 (Fukuoka, 1999), 585-592, Int. Soc. Anal. Appl. Comput. 7, Kluwer Acad. Publ., Dordrecht, 2000.

[21] L. Losonczi, Inequalities for integral mean values, J. Math. Anal. Appl. 61 (1977), no. $3,586-606$

[22] A. W. Marshall and I. Olkin, Inequalities: Theory of Majorization and Its Applications, New York, Academic Press, 1979.

[23] M. Merkle, Convexity, Schur-convexity and bounds for the gamma function involving the digamma function, Rocky Mountain J. Math. 28 (1998), no. 3, 1053-1066.

[24] C. P. Niculescu, Convexity according to the geometric mean, Math. Inequal. Appl. 3 (2000), no. 2, 155-167.

[25] E. Neuman and J. Sándor, Inequalities involving Stolarsky and Gini means, Math. Pannon. 14 (2003), no. 1, 29-44.

[26] E. Neuman and Zs. Páles, On comparison of Stolarsky and Gini means, J. Math. Anal. Appl. 278 (2003), no. 2, 274-284.

[27] Zs. Páles, Comparison of two variable homogeneous means, General inequalities, 6 (Oberwolfach, 1990), 59-70, Internat. Ser. Numer. Math., 103, Birkhäuser, Basel, 1992.

[28] F. Qi, A note on Schur-convexity of extended mean values, Rocky Mountain J. Math. 35 (2005), no. 5, 1787-1793. 
[29] F. Qi, J. Sándor, and S. S. Dragomir, Notes on the Schur-convexity of the extended mean values, Taiwanese J. Math. 9 (2005), no. 3, 411-420.

[30] J. Sándor, A note on the Gini means, Gen. Math. 12 (2004), no. 4, 17-21.

[31] _ The Schur-convexity of Stolarsky and Gini means, Banach J. Math. Anal. 1 (2007), no. 2, 212-215.

[32] M. Shaked, J. G. Shanthikumar, and Y. L. Tong, Parametric Schur convexity and arrangement monotonicity properties of partial sums, J. Multivariate Anal. 53 (1995), no. 2, 293-310.

[33] H. N. Shi, S. H. Wu, and F. Qi, An alternative note on the Schur-convexity of the extended mean values, Math. Inequal. Appl. 9 (2006), no. 2, 219-224.

[34] H.-N. Shi, Y.-M. Jiang, and W.-D. Jiang, Schur-convexity and Schur-geometrically concavity of Gini means, Comput. Math. Appl. 57 (2009), no. 2, 266-274.

[35] C. Stepniak, Stochastic ordering and Schur-convex functions in comparison of linear experiments, Metrika 36 (1989), no. 5, 291-298.

[36] S. Toader and G. Toader, Complementaries of Greek means with respect to Gini means, Int. J. Appl. Math. Stat. 11 (2007), no. 7, 187-192.

[37] B.-Y. Wang, Foundations of Majorization Inequalities, Beijing Normal Univ. Press, Beijing, China, 1990.

[38] Z.-H. Wang, The necessary and sufficient condition for S-convexity and S-geometrically convexity of Gini mean, J. Beijing Ins. Edu. (Natural Science) 2 (2007), no. 5, 1-3.

[39] Z.-H. Wang and X.-M. Zhang, Necessary and sufficient conditions for Schur convexity and Schur-geometrically convexity of Gini means, Communications of inequalities researching 14 (2007), no. 2, 193-197.

[40] W.-F. Xia, The Schur harmonic convexity of Lehmer means, Int. Math. Forum 4 (2009), no. 41, 2009-2015.

[41] W.-F. Xia and Y.-M. Chu, Schur-convexity for a class of symmetric functions and its applications, J. Inequal. Appl. 2009 (2009), Art. ID 493759, 15 pages.

[42] Zh.-H. Yang, Simple discriminances of convexity of homogeneous functions and applications, Gāoděng Shùxué Yánjiū (Study in College Mathematics) 4 (2004), no. 7, 14-19.

[43] — On the homogeneous functions with two parameters and its monotonicity, J. Inequal. Pure Appl. Math. 6 (2005), no. 4, Art. 101.

[44] - On the log-convexity of two-parameter homogeneous functions, Math. Inequal. Appl. 10 (2007), no. 3, 499-516.

[45] - On the monotonicity and log-convexity of a four-parameter homogeneous mean, J. Inequal. Appl. 2008 (2008), Art. ID 149286, 12 pages.

[46] Some monotonictiy results for the ratio of two-parameter symmetric homogeneous functions, Int. J. Math. Math. Sci. 2009 (2009), Art. ID 591382, 12 pages.

[47] _ Necessary and sufficient conditions for Schur convexity of the two-parameter symmetric homogeneous means, Appl. Math. Sci. (Ruse) 5 (2011), no. 64, 3183-3190.

[48] - The log-convexity of another class of one-parameter means and its applications, Bull. Korean Math. Soc. 49 (2012), no. 1, 33-47.

[49] X.-M. Zhang, Schur-convex functions and isoperimetric inequalities, Proc. Amer. Math. Soc. 126 (1998), no. 2, 461-470.

[50] — Geometrically Convex Functions, Hefei, An'hui University Press, 2004.

SYSTEM Division

Zhejiang Province Electric Power Test and Research Institute

Hangzhou, Zhejiang, 310014, P. R. China

E-mail address: yzhkm@163.com 